\title{
CORRESPONDENCE
}

DOI: $10.1038 / s 41467-018-04972-5$

\section{On the origin of molecular oxygen in cometary comae}

\author{
K.L. Heritier (10 1, K. Altwegg², J.-J. Berthelier ${ }^{3}$, A. Beth ${ }^{1}$, C.M. Carr (1D 1, J. De Keyser (1) 4, A.I. Eriksson ${ }^{5}$, \\ S.A. Fuselier ${ }^{6,7}$, M. Galand (10 1, T.I. Gombosi (10 ${ }^{8}$, P. Henri ${ }^{9}$, F.L. Johansson ${ }^{5}$, H. Nilsson ${ }^{10}$, M. Rubin (10) ${ }^{2}$, \\ C. Simon Wedlund ${ }^{11}$, M.G.G.T. Taylor ${ }^{12} \&$ E Vigren $^{3}$
}

Molecular oxygen was detected ${ }^{1}$ in the coma of $67 \mathrm{P}$ by the onboard Rosetta Orbiter Spectrometer for Ion and Neutral Analysis (ROSINA)-Double Focusing Mass Spectrometer (DFMS) ${ }^{2}$. It had a high volume mixing of about $1-10 \%$. Primordial $\mathrm{O}_{2}$ within the nucleus of comet 67P is compatible with the instrumental observations $^{1,3}$. Yao and Giapis ${ }^{4}$ proposed an alternative mechanism. The Eley-Rideal (ER) reactions of energetic watergroup ions with $\mathrm{Si} / \mathrm{Fe}$ oxides, or other minerals present on the surface of the nucleus, can generate $\mathrm{O}_{2}^{-}$. Yao and Giapis ${ }^{4}$ suggested that $\mathrm{O}_{2}^{-}$and energetic $\mathrm{O}_{2}$ (after photo-detachment) generated through ER reactions can be detected by ROSINA-DFMS and must be an important contributor of the $\mathrm{O}_{2}$ reported. In this correspondence paper, we do not disregard the fact that ER reactions can happen on the surface of the nucleus. However, we demonstrate that the amounts of $\mathrm{O}_{2}^{-}$produced through ER reactions cannot explain a significant fraction of the $\mathrm{O}_{2}$ detected by ROSINA-DFMS. In addition, we find that $\mathrm{O}_{2}^{-}$and energetic $\mathrm{O}_{2}$ generated through ER reactions could not be efficiently converted into the ROSINA-DFMS instrument and are therefore not responsible for the reported measurements.

To estimate the maximum amount of oxygen produced through ER reactions, we first consider the amount of ions, produced within the coma, that could impact the comet nucleus. Consistently with the multi-instrument analysis of Rosetta Plasma Consortium (RPC) and ROSINA sensors ${ }^{5,6}$, the number density of the expanding gas at a cometocentric distance $r$ is ${ }^{7}$ :

$$
n(r)=\frac{Q}{4 \pi u r^{2}}
$$

where $Q$ is the total outgassing rate and $u$, the outflow velocity. We assume a maximum global $Q$ to estimate the maximum amount of ions produced at a given time. The global ionization frequency is taken to be $v=10^{-6} \mathrm{~s}^{-1}$, which is an upper-bound based on measurements ${ }^{8}$. The production rate, $\mathrm{d} P$, of ions in each spherical shell of thickness $\mathrm{d} r$ is constant:

$$
\mathrm{d} P=\nu n(r) 4 \pi r^{2} \mathrm{~d} r=(\nu Q / u) \mathrm{d} r
$$

Once produced, ions retain the velocity of their parent neutrals for several kilometers ${ }^{5}$. Eventually these ions are picked up by the solar wind and have been observed moving anti-sunward ${ }^{9}$. The impact rate $Q^{*}$ onto the nucleus of radius $r_{0}(2 \mathrm{~km})$ for unidirectional motion can thus be estimated as:

$$
Q^{*}=\int_{r_{0}}^{\infty}\left(\frac{\pi r_{0}^{2}}{4 \pi r^{2}}\right) \frac{\nu Q}{u} \mathrm{~d} r=\frac{\nu Q r_{0}}{4 u}
$$

This calculation also holds for random motion of ions (Fig. 1). It is somewhat simplistic but still provides an order of magnitude of the number of ions hitting the nucleus. Furthermore, even coupled with a uniform circular gyration induced by the magnetic field, the amount of ions hitting a spherical target would remain the same. The farther away an ion is, the less-likely it is to return to the target. In addition, the presence of ambipolar electric fields to maintain ion-electron neutrality may repel ions approaching the nucleus ${ }^{10}$.

Let us assume that every collision leads to an ER reaction with $100 \%$ yield. The production rate of $\mathrm{O}_{2}^{-}$through this process is therefore directly approximated by $Q^{*}$. We assume a realistic (but slow, to obtain an upper bound) neutral outflow velocity of 500 $\mathrm{m} \mathrm{s}^{-1} \cdot Q^{*}$ can be related to $Q_{\mathrm{O}_{2}}$, the total production rate of $\mathrm{O}_{2}$ observed by ROSINA-DFMS, assuming low $\mathrm{O}_{2}$ mixing ratio (1\%) for our upper-bound estimation ${ }^{1}$ :

$$
Q^{*}=Q_{\mathrm{O}_{2}}^{\mathrm{ER}} \simeq 10^{-6} \mathrm{Q} \simeq 10^{-4} Q_{\mathrm{O}_{2}} \ll Q_{\mathrm{O}_{2}}
$$

\footnotetext{
${ }^{1}$ Department of Physics, Imperial College London, Prince Consort Road, London SW7 2AZ, UK. 2 Physikalisches Institut, University of Bern, Sidlerstrasse 5, 3012 Bern, Switzerland. ${ }^{3}$ LATMOS/IPSL-CNRS-UPMC-UVSQ, 94100 Saint-Maur, France. ${ }^{4}$ BIRA-IASB, Royal Belgian Institute for Space Aeronomy, Ringlaan 3, Brussels, Belgium. ${ }^{5}$ Swedish Institute of Space Physics, Ångström Laboratory, Lägerhyddsvägen 1, 75237 Uppsala, Sweden. ${ }^{6}$ Southwest Research Institute, P.O. Drawer 28510, San Antonio, TX 78228-0510, USA. ${ }^{7}$ University of Texas at San Antonio, San Antonio, TX 78249, USA. ${ }^{8}$ Department of Atmospheric, Oceanic and Space Sciences, University of Michigan, Ann Arbor, MI 48109, USA. ${ }^{9}$ LPC2E, CNRS, 3 Avenue de la recherche scientifique, 45071 Orléans, France. ${ }^{10}$ Swedish Institute of Space Physics, P.O. Box 812, 98128 Kiruna, Sweden. ${ }^{11}$ Department of Physics, University of Oslo, Sem Sæelands vei 24postbox 1048, 0317 Oslo, Norway. ${ }^{12}$ European Space Agency, ESTEC, Keplerlaan 1, Noordwijk 2200 AG, The Netherlands. Correspondence and requests for materials should be addressed to K.L.H. (email: k.heritier15@imperial.ac.uk)
} 
The maximum fraction of $\mathrm{O}_{2}^{-}$anions produced through ER reactions, followed by photo-detachment-process which is associated with small cross sections ${ }^{11}$-is therefore insignificant with respect to the observed $Q_{\mathrm{O}_{2}}{ }_{2}$. Furthermore, extrapolating the lab samples ${ }^{4}$ as surrogates of the cometary surface is highly speculative given the nucleus porosity $\left(30-65 \%^{12}\right)$ and the presence of $\mathrm{C}$ and $\mathrm{N}$ bearing refractory species ${ }^{13}$ within the surface composition.

An alternative approach is to compute the energetic ion fluxes that could potentially strike the nucleus. Nilsson et al. ${ }^{9}$ computed 24-h average time series of the cometary energetic ion fluxes measured by RPC-Ion Composition Analyzer (ICA) ${ }^{14}$ over the 2year mission. The maximum cometary energetic ion flux measured was about $10^{13} \mathrm{~m}^{-2} \mathrm{~s}^{-1}$. It leads to a total surface production rate $Q_{\mathrm{O}_{2}}^{E R}$ of $\mathrm{O}_{2}^{-}$of the order of $10^{20} \mathrm{~s}^{-1}$. It is still too low to reach volume mixing ratios of $1-10 \%$ : even for a low $Q_{\mathrm{O}_{2}}$ (1\% mixing ratio of $\mathrm{O}_{2}$ and low $Q$ of $\left.10^{26} \mathrm{~s}^{-1}\right)^{1,7}, Q_{\mathrm{O}_{2}}^{E R}$ is about $10^{-4} Q_{\mathrm{O}_{2}}$.

Figure 2 shows a time series of the energetic cometary watergroup ion fluxes measured by RPC-ICA close to the nucleus from 5 to 23 March 2016. After correction for the negative spacecraft potential measured by the Rosetta dual Langmuir Probe (RPC-LAP $)^{15}$, the flux is integrated over the $50-300 \mathrm{eV}$ energies that are efficient to trigger the ER reactions ${ }^{4}$. The maximum value is $10^{11} \mathrm{~m}^{-2} \mathrm{~s}^{-1}$. The number densities of $\mathrm{O}_{2}$ and $\mathrm{H}_{2} \mathrm{O}$ derived from ROSINA-DFMS and ROSINA-COPS are over-plotted in Fig. 2. The fluctuations are essentially seasonal ${ }^{16}$ with high outgassing over the summer hemisphere and low outgassing over the winter hemisphere. There is a clear anti-correlation between $\mathrm{O}_{2}$ densities and the energetic ion fluxes, drivers of ER reactions. With a high outgassing, the flux is weakened by the time it reaches the nucleus due to ion-neutral collisions. The highest neutral densities of $\mathrm{O}_{2}$ are therefore not driven by energetic ions through ER reactions on the surface. In addition, $\mathrm{O}_{2}$ and $\mathrm{H}_{2} \mathrm{O}$ are strongly correlated ${ }^{16}$ (Fig. 2) and follow a $r^{-2}$ Haser law $^{7}$. This is incompatible with an extended source of $\mathrm{O}_{2}$ through ER reactions that could happen on dust grains or the spacecraft itself.

As for the instrumental detections, Yao and Giapis ${ }^{4}$ claim that $\mathrm{O}_{2}^{-}$and energetic $\mathrm{O}_{2}$ may hit the gold-coated surfaces of the ROSINA-DFMS and be efficiently converted into $\mathrm{O}_{2}^{+}$. ROSINA-DFMS ${ }^{2}$ has two operation modes (neutral and ion) which differ by only the electrostatic bias and filament emission. In neutral mode, the ionization box is biased relative to spacecraft potential by $+200 \mathrm{~V}$ to keep primarily cometary ions out and the filament emits electrons to ionize incoming neutrals. In ion mode, ROSINA-DFMS primarily measures thermal ions with no bias voltage on the ionization box and the filament is heated below the electron emission threshold (sub-emission). The angular acceptance of the electrostatic analyser is $5^{\circ}$ and the energy acceptance is $1 \%$ of the nominal ion energy which depends on the mass measured. For mass $32 \mathrm{Da}$, this voltage is $-1900 \mathrm{~V}$ : only species with energies below $19 \mathrm{eV}$ pass the electrostatic analyser.

In neutral mode, $\mathrm{O}_{2}^{-}$anions with energies higher than the spacecraft potential (varying between 0 and $-30 \mathrm{~V}^{15}$ ) would enter the instrument and be accelerated to about $200 \mathrm{eV}$. The impact of the gold surface to convert into $\mathrm{O}_{2}^{+}$would have to be accompanied by the loss of the $200 \mathrm{eV}$ with minimal scattering to fit into the narrow angular acceptance angle. It is rather unlikely.

In the case of energetic $\mathrm{O}_{2}$, following the suggested conversion to $\mathrm{O}_{2}^{+}$via collisions with the gold surface, these ions, with energy below $19 \mathrm{eV}$ and minimal angular scattering $\left(<5^{\circ}\right)$, could enter the sensor equally well in neutral and ion modes. As such, the peak height for mass $32 \mathrm{Da}$ would be the same in both modes. In neutral high resolution mode, the peak was continuously strong, normally exceeding $10^{4}$ ions. However, in the ion mode, the signal was below detection limit for mass $32 \mathrm{Da}$ in high resolution. This precludes the possibility that $\mathrm{O}_{2}^{-}$and energetic $\mathrm{O}_{2}$ are efficiently ionized in the DFMS sensor by hitting the surfaces of the instrument. These observations are much more consistent with a thermal neutral $\mathrm{O}_{2}$ population, partially ionized ${ }^{8}$. If the ER mechanism is in action, its contribution to the $\mathrm{O}_{2}$ peak in the neutral mode is very minor.

\section{Conclusions}

We have used generous assumptions in terms of production rates and ion fluxes to assess the production of $\mathrm{O}_{2}^{-}$through ER reactions as a mechanism to explain Rosetta observations. Even with these assumptions, the amount of $\mathrm{O}_{2}$ produced is insignificant (by several orders of magnitude) with respect to what was detected by ROSINA-DFMS. There are not enough ions in the coma and the series of events required to trigger these processes are individually too rare. Furthermore, cometary ion fluxes are anti-correlated to the $\mathrm{O}_{2}$ densities observed by ROSINA. Finally, in terms of the instrument itself, there is little evidence of the production and detection of products $\mathrm{O}_{2}^{-}$and energetic $\mathrm{O}_{2}$ by ROSINA-DFMS. While ER reactions may occur, they cannot explain the amounts of $\mathrm{O}_{2}$

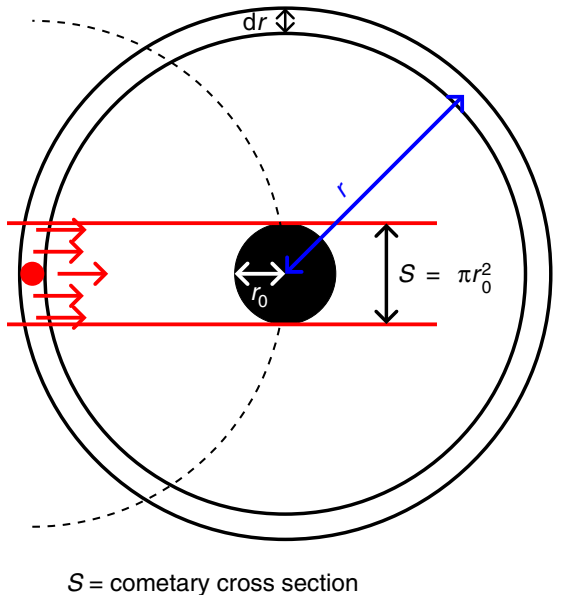

Fraction of ions crossing the comet $=\frac{S}{4 \pi r^{2}}=\left(\frac{r_{0}}{2 r}\right)^{2}$

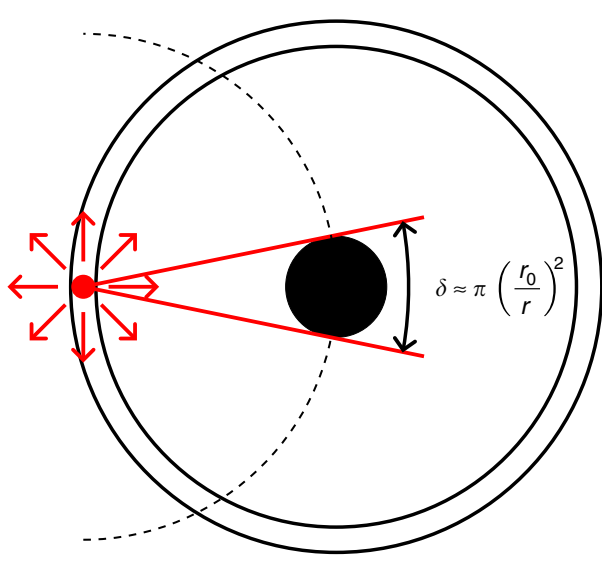

$\delta$ [str]: cometary solid angle seen by the ion

Probability to hit the comet $\approx \frac{\delta}{4 \pi}=\left(\frac{r_{0}}{2 r}\right)^{2}$

Fig. 1 Schematic of the ion motion and their probability of returning to the nucleus. Shown for unidirectional motion (left side) and random motion (right side). In both case, the probability for an ion (red dot), created at $r$, to return to the nucleus is $\left(\frac{r_{0}}{2 r}\right)^{2}$ 


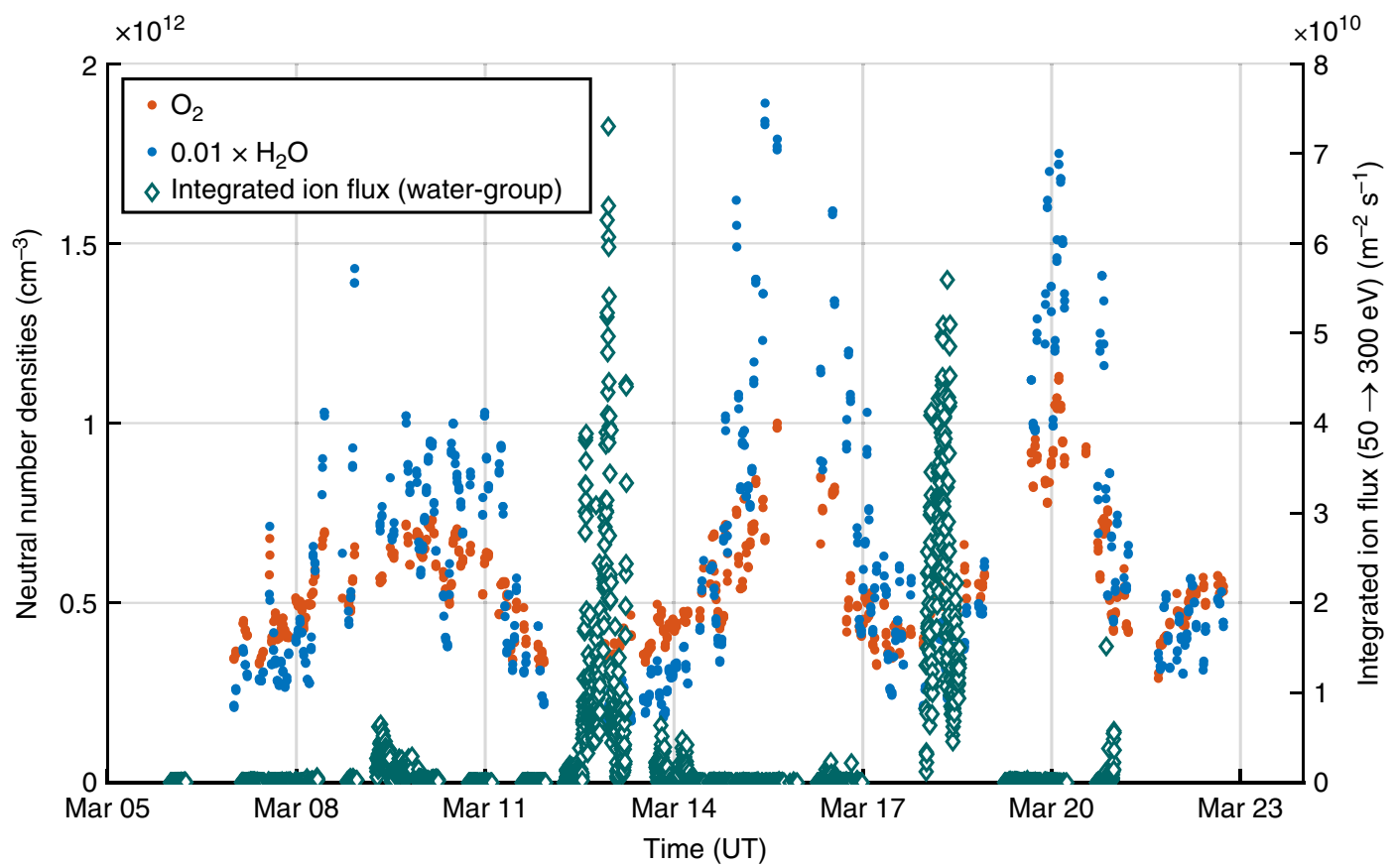

Fig. 2 Time series of oxygen and water neutral number densities. Time series of the $\mathrm{O}_{2}$ (orange) and $\mathrm{H}_{2} \mathrm{O}$ (blue) neutral number densities (left $y$-axis) from March 6 to 23, 2016. The spacecraft was located at about $10 \mathrm{~km}$ from the nucleus. Note that the $\mathrm{H}_{2} \mathrm{O}$ neutral densities have been multiplied by $10^{-2}$ to fit on this scale. Time series of the total RPC-ICA energetic water-group ions $\left(\mathrm{H}_{2} \mathrm{O}^{+}, \mathrm{H}_{3} \mathrm{O}^{+}, \mathrm{OH}^{+}\right)$of cometary origin (green), corrected for the RPC-LAP spacecraft potential ${ }^{15}$ and integrated over the effective energy range of the Eley-Rideal reactions (50-300 eV) ${ }^{4}$ (right $y$-axis)

detected. Primordial $\mathrm{O}_{2}{ }^{1,3}$ remains compatible with the quantities and trends of molecular oxygen measured by ROSINA-DFMS, while other theories ${ }^{17}$ discuss other plausible sources.

Received: 28 July 2017 Accepted: 5 June 2018

Published online: 03 July 2018

\section{References}

1. Bieler, A. et al. Abundant molecular oxygen in the coma of comet $67 \mathrm{P} /$ Churyumov-Gerasimenko. Nature 526, 678-681 (2015).

2. Balsiger, H. et al. Rosina-Rosetta Orbiter Spectrometer for Ion and Neutral Analysis. Space Sci. Rev. 128, 745-801 (2007).

3. Taquet, V., Furuya, K., Walsh, C. \& van Dishoeck, E. F. A primordial origin for molecular oxygen in comets: a chemical kinetics study of the formation and survival of $\mathrm{O}_{2}$ ice from clouds to discs. Mon. Not. R. Astron. Soc. 462, S99-S115 (2016).

4. Yao, Y. \& Giapis, K. P. Dynamic molecular oxygen production in cometary comae. Nat. Commun. 8, 15298 (2017).

5. Galand, M. et al. Ionospheric plasma of comet $67 \mathrm{P}$ probed by Rosetta at 3 au from the Sun. Mon. Not. R. Astron. Soc. 462, S331-S351 (2016).

6. Heritier, K. L. et al. Vertical structure of the near-surface expanding ionosphere of comet 67P probed by Rosetta. Mon. Not. R. Astron. Soc. 469, S118-S129 (2017).

7. Hansen, K. C. et al. Evolution of water production of $67 \mathrm{P} /$ ChuryumovGerasimenko: an empirical model and a multi-instrument study. Mon. Not. R. Astron. Soc. 462, S491-S506 (2016).

8. Heritier, K. L. et al. Ion composition at comet $67 \mathrm{P}$ near perihelion: Rosetta observations and model-based interpretation. Mon. Not. R. Astron. Soc. 469, S427-S442 (2017).

9. Nilsson, H. et al. Evolution of the ion environment of comet 67P during the Rosetta mission as seen by RPC-ICA. Mon. Not. R. Astron. Soc. 469, S252-S261 (2017).

10. Deca, J. et al. Electron and ion dynamics of the solar wind interaction with a weakly outgassing comet. Phys. Rev. Lett. 118, 205101 (2017).

11. Lee, L. C. \& Smith, G. P. Photodissociation and photodetachment of molecular negative ions. VI. Ions in $\mathrm{O}_{2} / \mathrm{CH}_{4} / \mathrm{H}_{2} \mathrm{O}$ mixtures from 3500 to 8600 . J. Chem. Phys. 70, 1727-1735 (1979).
12. Lethuillier, A. et al. Electrical properties and porosity of the first meter of the nucleus of 67P/Churyumov-Gerasimenko. As constrained by the Permittivity Probe SESAME-PP/Philae/Rosetta. Astron. Astrophys. 591, A32 (2016).

13. Quirico, E. et al. Refractory and semi-volatile organics at the surface of comet 67P/Churyumov-Gerasimenko: Insights from the VIRTIS/Rosetta imaging spectrometer. Icarus 272, 32-47 (2016).

14. Nilsson, H. et al. RPC-ICA: the ion composition analyzer of the rosetta plasma consortium. Space Sci. Rev. 128, 671-695 (2007).

15. Odelstad, E. et al. Measurements of the electrostatic potential of Rosetta at comet 67P. Mon. Not. R. Astron. Soc. 469, S568-S581 (2017).

16. Fougere, N. et al. Direct simulation Monte Carlo modelling of the major species in the coma of comet 67P/Churyumov-Gerasimenko. Mon. Not. R. Astron. Soc. 462, S156-S169 (2016).

17. Dulieu, F., Minissale, M. \& Bockele-Morvan, D. Production of $\mathrm{O}_{2}$ through dismutation of $\mathrm{H}_{2} \mathrm{O}_{2}$ during water ice desorption: a key to understanding comet $\mathrm{O}_{2}$ abundances. Astron. Astrophys. 597, A56 (2017).

\section{Author contributions}

H.N., A.I.E., P.H., and C.M.C., as Principal Investigators of RPC; K.A., as Principal Investigator of ROSINA; and M.G.G.T., as Rosetta Project Scientist, initiated the project to write a correspondence paper on this topic, based on Rosetta in-situ measurements. K. L.H. coordinated the team effort and carried out the manuscript preparation. K.A., J.-J.B., C.M.C., J.D.K., A.I.E., S.A.F., M.G., T.I.G., P.H., F.L.J., H.N., M.R., C.S.W., M.G.G.T., and E.V. contributed to the manuscript preparation. K.L.H., M.G., and A.B. computed an upper-bound for the ion-nucleus collisions. C.S.W. and H.N. analyzed the RPC-ICA dataset and derived the energetic ion fluxes used in Fig. 2. F.L.J. and A.I.E. analyzed the RPC-LAP dataset used to correct the ion fluxes for the spacecraft potential. K.A. and M. R. analyzed the ROSINA-DFMS dataset shown in Fig. 2. C.M.C., A.I.E., P.H. and H.N contributed to the development and operation of the RPC sensors. K.L.H., A.B., A.I.E., M.G., P.H., F.L.J., H.N., C.S.W. and E.V. contributed to the interpretation of the RPC dataset. K.A., M.R., J.-J.B., J.D.K., S.A.F. and T.I.G. contributed to the development and operation of the ROSINA-DFMS instrument and to the interpretation of its dataset.

\section{Additional information}

Competing interests: The authors declare no competing interests.

Reprints and permission information is available online at http://npg.nature.com/ reprintsandpermissions/ 
Publisher's note: Springer Nature remains neutral with regard to jurisdictional claims in published maps and institutional affiliations.

(c) (i) Open Access This article is licensed under a Creative Commons Attribution 4.0 International License, which permits use, sharing, adaptation, distribution and reproduction in any medium or format, as long as you give appropriate credit to the original author(s) and the source, provide a link to the Creative Commons license, and indicate if changes were made. The images or other third party material in this article are included in the article's Creative Commons license, unless indicated otherwise in a credit line to the material. If material is not included in the article's Creative Commons license and your intended use is not permitted by statutory regulation or exceeds the permitted use, you will need to obtain permission directly from the copyright holder. To view a copy of this license, visit http://creativecommons.org/ licenses/by/4.0/.

(c) The Author(s) 2018 